\title{
Low incidence of prostate cancer identified in the transition and anterior zones with transperineal biopsy
}

This article was published in the following Dove Press journal:

Research and Reports in Urology

4 December 2012

Number of times this article has been viewed

\author{
Teresa L Danforth' \\ K Kent Chevli ${ }^{1,2}$ \\ Louis Baumann ${ }^{1,2}$ \\ Michael Duff 1,2
}

'The State University of New York (SUNY), Buffalo, NY, ${ }^{2}$ Cancer Care of Western New York, Cheektowaga, NY, USA

Correspondence: Michael Duff Cancer Care of Western New York, 3085 Harlem Rd, Cheektowaga, NY 14225, USA

Tel + I 7168445500

Fax +I 7168445550

Email mduff@cancercarewny.com
Purpose: Determine the incidence of anterior (AZ) and transition (TZ) zone prostate cancers using a transperineal mapping approach.

Methods: A retrospective review of 137 patients with history of previous negative biopsy undergoing transperineal saturation biopsy for an elevated prostate-specific antigen (PSA), high-grade prostate intraepithelial neoplasia, atypical small acinar proliferation history, or abnormal digital rectal exam. The number of biopsy cores was determined by prostate volume and obtained using a predefined template. The electronic medical records were reviewed for patients' clinical and pathological characteristics.

Results: Forty-one of 137 patients (31.4\%) had positive biopsy for prostate adenocarcinoma; 11 were from 24-core, 19 from 36-core, and 11 from 48-core sampling. Glands $>45 \mathrm{~mL}$ had a mean of 1.7 previous biopsies and a PSA of $9.1 \mathrm{ng} / \mathrm{mL}$. Glands $<30 \mathrm{~mL}$ were 1.3 and $6.3 \mathrm{ng} / \mathrm{mL}$ and glands $30-45 \mathrm{~mL}$ were 1.4 and $6.5 \mathrm{ng} / \mathrm{mL}$. Glands $<45 \mathrm{~mL}$ had a higher number of positive biopsies per total cores. Seven patients chose active surveillance while 34 chose treatment. Of the 36- and 48-cores biopsies, $2.2 \%$ and $1.5 \%$, respectively, were positive in the TZ. One patient was AZ-positive, 1 was TZ-positive, and 18 were peripheral zone (PZ)-positive alone. Twelve patients had cancer detected in PZ and TZ. Two patients developed urinary retention and one had a urine infection.

Conclusion: Transperineal saturation biopsy is a safe and efficacious method of prostate cancer detection in patients with previous negative biopsy and high suspicion for cancer. Few cancers were found to originate in the $\mathrm{TZ}$ or $\mathrm{AZ}$ alone. We recommend that initial biopsy templates should sample PZ with less focus on the TZ.

Keywords: carcinoma, prostate, biopsy, transperineal

\section{Introduction}

An ultrasound-guided transrectal approach has become the standard biopsy technique for patients who choose to undergo further evaluation based on age, rectal exam and prostate-specific antigen (PSA) kinetics. A parasagittal sextant pattern may be adequate; however, recent series have suggested an extended-core biopsy protocol to improve cancer detection. ${ }^{1,2}$ This may be especially useful in those patients with at least one previous negative biopsy. ${ }^{3-8}$ This has led to different biopsy patterns to explore the need for additional biopsies both in the transitional zone (TZ) and lateral zones of the prostate.

Sampling the entire gland has been termed a saturation biopsy which has been shown to increase detection rates of prostate cancer after a previous negative biopsy with rates ranging from $11.1 \%-34 \% .^{3-8}$ Chen et al used computer modeling of 
prostatectomy specimens and concluded that the areas of the prostate not sampled on routine biopsy include the anterior TZ, midline of the peripheral zone (PZ) and anterior-inferior horn of the PZ. ${ }^{1}$ Various perineal biopsy templates have been described that incorporate sampling of these specific zones of the prostate. ${ }^{9-16}$

The use of TZ and anterior zone (AZ) biopsies or midline biopsies to aid in the detection of clinically significant cancer has been debated. The incidence of cancers arising in the $\mathrm{TZ}$ of the prostate has been estimated to be $2 \%-30 \%{ }^{17-26}$ Little information is available on the incidence of cancers arising in the AZ, with Terris et al reporting a low incidence of $1.8 \% .{ }^{27}$ Even in studies with a low incidence of TZ-only prostate cancers, saturation biopsies have been shown to improve detection rates of cancer up to $13 \%$ in select patient populations. ${ }^{19}$ This may be applicable only to those men with certain clinical characteristics including multiple previous negative biopsies or those with large prostates. ${ }^{19}$

The use of the transperineal biopsy approach allows for systematic mapping of the prostate gland with potential access to zones of the prostate not easily accessible using the transrectal technique. This technique typically requires general anesthesia. It has been shown to be safe and adverse events are rarely reported. ${ }^{9-14}$

In this study, we retrospectively examined the clinical and pathology data from 137 patients who underwent an ultrasound-guided transperineal saturation biopsy. Our template included the AZ, TZ, and lateral zones of the prostate. We correlated the positive biopsy specimens with specific anatomic locations within the prostate gland.

\section{Patients and methods}

During 2009 and 2010, 137 patients from our group underwent a transperineal saturation biopsy. Most patients underwent a previous negative biopsy for a persistently elevated PSA or abnormal digital rectal exam (DRE). Some patients had persistent abnormalities and a previous diagnosis of high-grade prostate intraepithelial neoplasia (HGPIN). The electronic medical records of these patients were reviewed retrospectively and 43 biopsies revealed a diagnosis of prostate cancer. Clinical and pathological data from these 43 charts were reviewed. We correlated the location of the positive cores with specific anatomic locations within the prostate gland that were defined a priori in our biopsy scheme. We also evaluated the impact of age, race, number of previous biopsies, previous PSA values, history of HGPIN or atypical small acinar proliferation (ASAP) on previous biopsy, DRE, family history, current PSA, prostate volume and Gleason scores on our findings. A positive family history was defined as a first- or second-degree relative with history of prostate cancer. Two patients were excluded from the study for incomplete pathological data. Study approval was obtained from the institutional review board of the State University of New York Buffalo. The study was conducted in accordance with GCP, the Declaration of Helsinki and all applicable regulations.

\section{Biopsy protocol}

The transperineal saturation biopsies were performed using general anesthesia and all patients underwent medical clearance prior to the procedure. Patients were instructed to discontinue anticoagulants 7 days prior to their procedure. Patients taking clopidogrel or warfarin were cleared by cardiologists to temporarily discontinue these medications and blood was drawn to measure the prothrombin time immediately prior to the procedure. Tamulosin $0.4 \mathrm{mg}$ daily was started one day prior to the procedure and continued for 2 weeks after the procedure unless the patients were previously on an alpha blocker. Ciprofloxacin $500 \mathrm{mg}$ twice per day was started 1 day prior the procedure and continued for a total of 8 days. Intravenous ciprofloxacin was given at the time of the procedure.

Once anesthetized, patients were placed in the dorsal lithotomy position and prepped and draped in the standard surgical fashion. Aerated lidocaine gel was injected into the urethra and a penile clamp was placed. A BK Medical (Peabody, MA) Falcon, Model 2101 ultrasound machine with a 7.5 MHz Model 8658 (BK Medical) probe was inserted into the rectum and stabilized using a RTP 6000 brachytherapy stabilizer and STP 110 precision stepper (North American Scientific, Pittsburgh, PA). The prostate volume was measured via a height-timeswidth-times-length technique and recorded in the chart. The appropriate transperineal biopsy guide was chosen based on the volume of the prostate (Figure 1) which indicates the number of positive cores and their location. Glands measuring less than $30 \mathrm{~mL}$ underwent a 24-core biopsy. Glands measuring 30-45 mL underwent a 36-core biopsy, and glands measuring greater than $45 \mathrm{~mL}$ underwent a 48-core biopsy. Biopsy samples were taken using an 18-gauge Bard Biopsy Systems (Tempe, AZ) Max-Core ${ }^{\circledR}$ disposable core biopsy instrument. Needles were guided to the appropriate position using a perineal template used for prostate brachytherapy procedures. Each biopsy specimen was placed in a separately labeled jar with the corresponding numbered location on the biopsy template, 1-24, 36, or 48. The specimens were then sent for pathology review. Specimens were graded as having either 

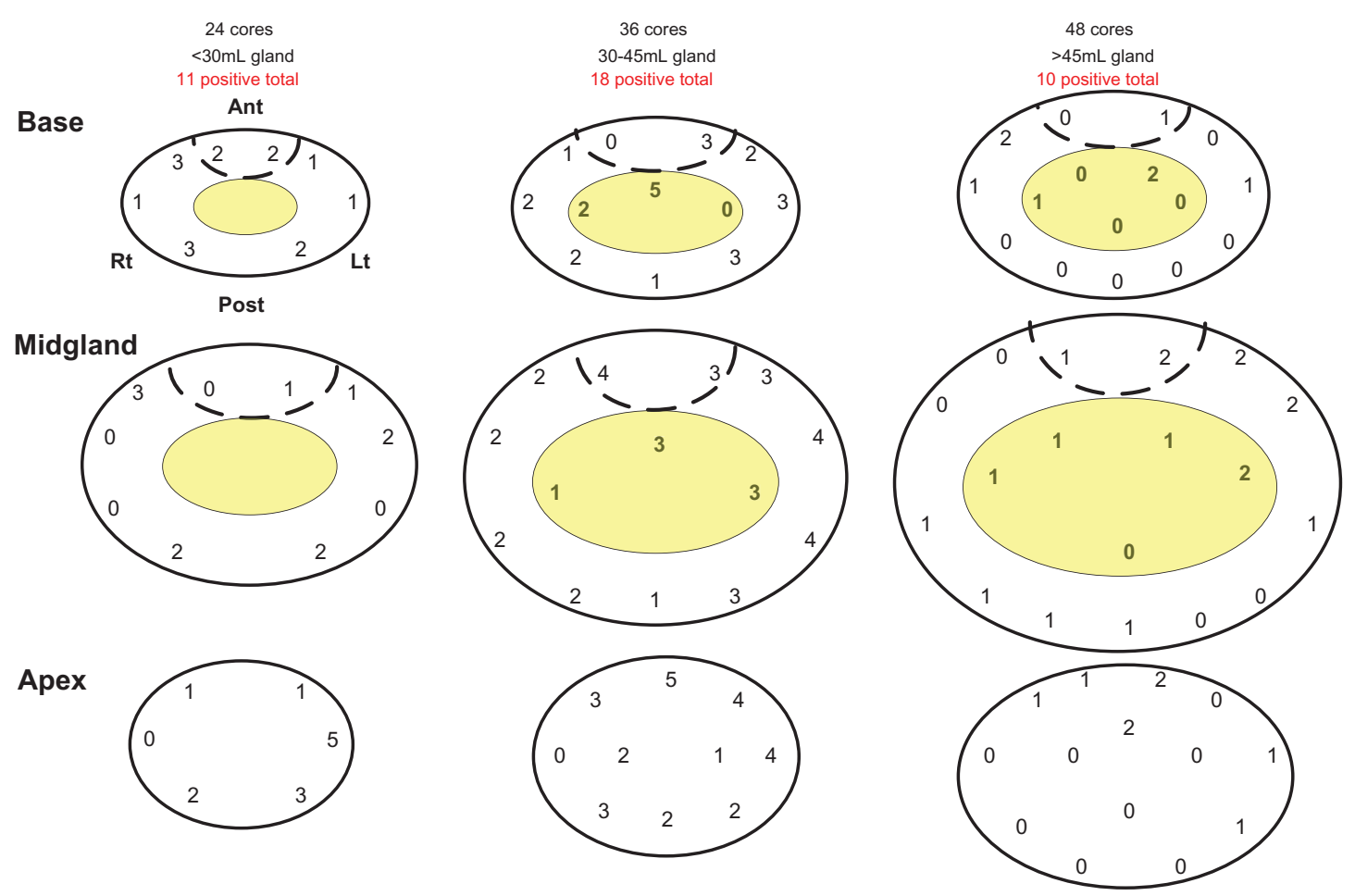

Figure I Transperineal prostate biopsy positive cores under anesthesia anatomic location guide.

Notes: Dotted line differentiates anterior zone of the prostate.

cancer, HGPIN, ASAP, or no cancer. All cores with cancer specified the histology, Gleason grade, and percentage of core involved. Each specimen positive for cancer was independently reviewed by two pathologists.

\section{Results}

A total of 137 patients underwent a transperineal prostate biopsy. Forty-three were positive for adenocarcinoma (31.4\%). Clinical characteristics of the 41 patients with complete pathologic data are summarized in Table 1. Eleven, 19 , and 11 patients with a positive biopsy underwent $24-$, 36-, and 48-core biopsies, respectively. All but four patients had undergone a previous transrectal guided prostate biopsy. These four patients required anesthesia for their initial biopsy due to inability to tolerate the procedure with local anesthesia.

Clinical characteristics are listed in Table 1. The average number of previous transrectal biopsies was higher in those with larger glands $(1.7 \pm 0.8$ for glands $>45 \mathrm{~mL})$ than smaller glands $(1.3 \pm 0.9$ for $<30 \mathrm{~mL}$ and $1.4 \pm 0.9$ for $30-45 \mathrm{~mL}$ ). The patients with glands $>45 \mathrm{~mL}$ had an average PSA value of $9.1 \pm 6.0 \mathrm{ng} / \mathrm{mL}$, which was also higher than those with either $<30 \mathrm{~mL}$ glands $(6.3 \pm 3.8 \mathrm{ng} / \mathrm{mL})$ or $30-45 \mathrm{~mL}(6.5 \pm 3.8 \mathrm{ng} / \mathrm{mL})$. Twenty-five patients $(60.1 \%)$ had a history of HGPIN or ASAP on previous biopsy. Twelve patients (29.3\%) had clinical T2 disease. Eight patients who underwent a 36-core biopsy had a positive family history of prostate cancer, whereas zero patients who underwent a 24-core and four patients who underwent a 48-core biopsies had a positive family history. Patients who underwent 24 - and 36-core biopsy had overall higher number of positive biopsies per total cores than 48 -core patients $(14.8 \%$ and $13.0 \%$ vs $7.6 \%$ ). No patients who underwent a metastatic workup had positive findings.

Seven patients chose to undergo active surveillance for low-risk disease. The other 34 patients chose to undergo some type of active treatment, which included intensitymodulated radiation therapy (IMRT) with or without androgen-deprivation therapy (ADT), ADT alone, or radical prostatectomy. Table 2 shows the distribution of the positive prostate biopsy cores. Overall, the percentage of positive biopsies decreased as the prostate size increased with $14.8 \%, 13.0 \%$, and $7.6 \%$ positive rates in $24-, 36-$, and 48-core biopsies respectively. Most of the positive cores were detected in the $\mathrm{PZ}$ and $\mathrm{AZ}$ with few positive biopsies in the TZ, $2.2 \%$ and $1.5 \%$ in the 36 - and 48 -core biopsies, respectively. One (2.4\%), one (2.4\%), and 18 (44.9\%) patients had cancers detected in the $\mathrm{AZ}, \mathrm{TZ}$, and $\mathrm{PZ}$ only, respectively. Twelve (29.3\%) patients had cancer detected both in the TZ and PZ. 
Table I Biopsy data

\begin{tabular}{|c|c|c|c|c|}
\hline & 24-core $(n=I I)$ & 36 -core $(n=19)$ & 48 -core $(n=I I)$ & Overall $(n=4 I)$ \\
\hline Average age, years (range) & $68(59-82)$ & 69 (59-82) & $73(65-82)$ & 70 (59-82) \\
\hline \multicolumn{5}{|l|}{ Race } \\
\hline Caucasian (\%) & $9(82)$ & $19(100)$ & II (100) & $39(95)$ \\
\hline African American (\%) & $2(18)$ & $0(0)$ & $0(0)$ & $2(5)$ \\
\hline Average number of previous biopsies \pm SD & $1.3 \pm 0.9$ & $1.4 \pm 0.9$ & $1.7 \pm 0.79$ & $1.4 \pm 0.87$ \\
\hline Average PSA at last biopsy $(\mathrm{ng} / \mathrm{mL}) \pm \mathrm{SD}$ & $6.3 \pm 3.8$ & $6.5 \pm 3.8$ & $9.1 \pm 6.0$ & $7.2 \pm 4.6$ \\
\hline Previous (\%) HGPIN/ASAP & $5(45)$ & $13(68)$ & $7(63.6)$ & $25(61.0)$ \\
\hline cT2 (\%) & $2(18)$ & $8(42)$ & $2(18.1)$ & $12(29.2)$ \\
\hline Positive Fam Hx & $0(0)$ & $8(42)$ & $4(36.3)$ & $12(29.2)$ \\
\hline Average prebiopsy PSA $(\mathrm{ng} / \mathrm{mL}) \pm S D$ & $7.9 \pm 6.0$ & $7.9 \pm 5.5$ & $10.4 \pm 9.0$ & $8.6 \pm 6.6$ \\
\hline Prostate volume $(\mathrm{mL})$ & $27.9 \pm 5.4$ & $39.8 \pm 4.8$ & $58.0 \pm 5.6$ & $41.5 \pm 12.4$ \\
\hline Average number of positive cores $\pm S D$ & $3.5 \pm 2.8$ & $4.7 \pm 4.7$ & $3.6 \pm 2.5$ & $4.1 \pm 3.7$ \\
\hline \multicolumn{5}{|l|}{ Treatment } \\
\hline Active treatment (\%) & $9(8 \mid .2)$ & $17(89.5)$ & $8(72.7)$ & $34(82.9)$ \\
\hline Active surveillance (\%) & $2(18.2)$ & $2(10.5)$ & $3(27.2)$ & $7(17.1)$ \\
\hline
\end{tabular}

Abbreviations: ASAP, atypical small acinar proliferation; CT2, stage Il cancer; Fam Hx, family history; HGPIN, high-grade prostate intraepithelial neoplasia; PSA, prostatespecific antigen; SD, standard deviation.

Three patients who underwent 48-core biopsies had complications (7.3\%). Two patients developed urinary retention requiring catheterization for 2 and 12 days, respectively. One patient was hospitalized for prostatitis postoperatively and made a full recovery. No patients who underwent 24- or 36-cores biopsies had postoperative complications.

\section{Discussion}

It has been shown that saturation biopsy is a useful tool in patients with an elevated risk for cancer and a previous negative biopsy. However, saturation biopsy does not appear to improve detection in patients as an initial biopsy technique. ${ }^{28}$ Many studies suggest the need for extended biopsy technique should be reserved for high-risk patients in whom previous biopsies have been negative and tailored to their age, clinical stage, and volume of the prostate. ${ }^{29}$

Multiple templates have been suggested when performing saturation biopsy including transrectal and transperineal approaches, with local or general anesthesia. Emiliozzi et al

Table 2 Zone distribution of biopsies

\begin{tabular}{|c|c|c|c|c|}
\hline & $\begin{array}{l}24 \text {-core } \\
(n=I I)\end{array}$ & $\begin{array}{l}36-\text { core } \\
(n=19)\end{array}$ & $\begin{array}{l}48 \text {-core } \\
(n=I I)\end{array}$ & Overall \\
\hline Total cores & 264 & 684 & 528 & \\
\hline Total positive cores (\%) & $39(14.8)$ & $89(13.0)$ & $40(7.6)$ & \\
\hline PZ-positive cores (\%) & $33(12.5)$ & $64(9.4)$ & $28(5.3)$ & \\
\hline AZ-positive cores (\%) & $6(2.3)$ & $10(1.5)$ & $4(0.8)$ & \\
\hline TZ-positive cores (\%) & & I5 (2.2) & $8(1.5)$ & \\
\hline AZ-only patients (\%) & I (9.1) & $0(0)$ & $0(0)$ & I (2.4) \\
\hline TZ-only patients (\%) & & I (5.3) & $0(0)$ & I (2.4) \\
\hline PZ-only patients (\%) & $5(45.5)$ & $8(4.2)$ & $5(45.5)$ & $18(44.9)$ \\
\hline TZ + PZ patients (\%) & & 7 (36.8) & $5(45.5)$ & $12(29.3)$ \\
\hline
\end{tabular}

Abbreviations: $A Z$, anterior zone; $P Z$, peripheral zone; $T Z$, transitional zone. as well as Vis et al compared cancer detection rates with transperineal and transrectal biopsies. Both demonstrated a higher detection rate with transperineal biopsy. ${ }^{15,16}$ Our template was created based on multiple different published templates used in the past to allow for adequate sampling of $\mathrm{TZ}, \mathrm{PZ}$, and AZ. These techniques allow for sampling of those areas not typically biopsied in a transrectal guided prostate biopsy. The biopsies were systematically taken, which allowed for the most precise location of tissue. The overall prostate cancer detection rate using transperineal biopsy in various risk populations is between $38 \%$ and $46 \%$, which is slightly higher than our detection rate at $31 \% .{ }^{9-16}$

Various studies have reported the importance of sampling the lateral zones of the prostate for increased detection rates..$^{30}$ The use of midline biopsies has been debated with detection rates of TZ-only prostate cancer ranging from $1.5 \%-28 \%$ depending on the population studied. ${ }^{17,22} \mathrm{TZ}$ biopsies have been found to be more useful in patients with previous negative biopsy, but have failed to significantly increase cancer detection rates on initial biopsy. ${ }^{17,21,24-27} \mathrm{We}$ found only one patient $(2.4 \%)$ with TZ-only cancer, which is consistent with the low rates found in previous studies of patients with previous negative biopsies.

Certain patient populations that may benefit from TZ biopsies are those with larger prostates and a previous negative biopsy with a persistently elevated PSA that is unexplained by a larger gland alone. Chang et al reported increased detection rate of $13 \%$ in patients with prostates greater than $50 \mathrm{~mL}$ and there was upgrading of Gleason grade in $14 \% .{ }^{19}$ Abdel-Khalek et al also reported improved cancer detection rates up to $14 \%$ in patients with previous negative biopsy 
and PSA $>10 \mathrm{ng} / \mathrm{dL} .{ }^{20}$ Both studies showed a low overall sensitivity of TZ biopsies of $55 \%$ and $61 \%$, indicating that most cancers will arise outside of the TZ with many never reaching the TZ. Our results show TZ-only cancers were found in only one patient (2.4\%) and an overall positivity in $34 \%$ of cancers identified, which is lower than seen in previous studies.

Few studies have looked at the incidence of AZ biopsies of the prostate. Terris et al showed that patients undergoing routine initial transrectal biopsy of the prostate had a low incidence of AZ-only cancers with only one of 55 patients $(1.8 \%) .{ }^{27}$ These results are consistent with ours in which only one patient in our study had AZ only cancer identified, who had one of 24 cores positive.

There are inherent risks with a prostate biopsy through the perineum including bleeding, urinary retention, and the risks of general anesthesia, though these risks are small. ${ }^{10}$ Merrick et al reported urinary retention rates up to $39.4 \%$ at day zero, but only $1.6 \%$ remained by day 6 with increased risks seen in patients with larger prostates. ${ }^{10}$ Our study shows a low rate of urinary retention at $7.3 \%$ (two patients). Both of these patients underwent 48 -core biopsies. Our low retention rates may be partly due to the prophylactic use of tamsulosin in all of our patients to try to avoid need for catheterization. Hematuria requiring catheterization ranged between $0 \%$ and 2.3\%. ${ }^{9,11-14}$ No significant bleeding events occurred in our study. All but four patients had undergone a previous transrectal-guided prostate biopsy. It was noted that these patients required anesthesia for their initial biopsy due to their inability to tolerate the procedure. The transperineal approach was at the discretion of the managing physician and may have been due to patient anatomy and gland volume.

Other series have attempted to focus on the location of prostate cancer within the gland by using radical prostatectomy specimens or magnetic resonance imaging scans. Falzarano et al reported on 72 patients who underwent a saturation biopsy prior to a radical prostatectomy. ${ }^{31}$ They found that prostate cancer was largely multifocal. Several patients in their series with unilateral cancer on saturation biopsy were found to have more extensive disease on radical prostatectomy specimen. Similarly, data from Korea identified a poor correlation with prostate cancer location using both MRI and standard prostate biopsy compared with radical prostatectomy specimen. ${ }^{32}$ Our study is somewhat different in that we took a transperineal approach to ensure adequate sampling of the gland while the others used a transrectal approach. It should, however, be noted that we do not have correlation with surgical specimens. There have been direct comparisons between the transperineal and the transrectal approaches. Kawakami et al compared a 12-core transrectal sampling with a 14-core transperineal sampling. They did not find a statistical difference in cancer detection rates. ${ }^{33} \mathrm{An}$ earlier study from Italy did, however, note a higher detection rate with a transperineal approach than with a transrectal approach. ${ }^{15}$ These data show that the transperineal approach is at least equivalent and may be superior to the transrectal technique. This is consistent with our data. We showed that in the areas that may not be adequately sampled with a transrectal approach there were very few additional cancers identified.

One limitation in this study is the small sample size, though our sample size is comparable to most previous studies of trans-perineal biopsy. With a total of 137 patients biopsied, 43 patients were found to have cancer. All but four patients had undergone a previous transrectal prostate biopsy. One of these patients when biopsied with the above protocol was found to have a TZ-only cancer. If the initial biopsy was done with the standard 12-core protocol, this patient would likely have required a repeat biopsy in the future, and potentially a saturation biopsy.

\section{Conclusion}

Transperineal saturation biopsy of the prostate is a safe and efficacious method of prostate cancer detection in patients with previous negative biopsy and high clinical suspicion for cancer. Few cancers are found to originate in the $\mathrm{TZ}$ or $\mathrm{AZ}$ alone, even in a subset of patients who have undergone previous biopsy. Initial biopsy templates should be aimed toward adequate sampling of the $\mathrm{PZ}$ with less focus on the TZ.

\section{Disclosure}

None of the authors of this paper report any conflicts of interest.

\section{References}

1. Chen ME, Troncoso P, Johnston DA, et al. Optimization of prostate biopsy strategy using computer based analysis. J Urol. 1997;158(6):2168-2175.

2. Guidchard G, Larre S, Gallina A, et al. Extended 21-sampling needle biopsy protocol for diagnosis of prostate cancer in 1000 consecutive patients. Eur Urol. 2007;52(2):430-435.

3. Stewart CS, Leibovich BC, Weaver AL, et al. Prostate cancer diagnosis using a saturation needle technique after previous negative sextant biopsies. J Urol. 2001;166(1):86-92.

4. Sajadi KP, Kim T, Terris MK, et al. High yield of saturation prostate biopsy for patients with previous negative biopsies and small prostates. Urology. 2007;70(4):691-695.

5. Fleshner N, Klotz L. Role of "saturation biopsy" in the detection of prostate cancer among difficult diagnostic cases. Urology. 2002;60(1):93-97. 
6. Rabets JC, Jones JS, Patel A, et al. Prostate cancer detection with office based saturation biopsy in a repeat biopsy population. J Urol. 2004; 172(1):94-97.

7. Borboroglu PG, Comer SW, Riffenburgh RH, et al. Extensive repeat transrectal ultrasound guided prostate biopsy in patient with previous benign sextant biopsies. J Urol. 2000;163(1):158-162.

8. Stav K, Leibovici D, Sandbank J, et al. Saturation prostate biopsy in high risk patients after multiple previous negative biopsies. Urology. 2008; 71(3):399-403.

9. Igel TC, Knight MK, Young PR, et al. Systematic transperineal ultrasound guided template biopsy of the prostate in patients at high risk. $J$ Urol. 2001;165(5):1575-1579.

10. Merrick GS, Taubenslag W, Andreini H, et al. The morbidity of transperineal template-guided prostate mapping biopsy. BJU Int. 2008; 101(12):1524-1529.

11. Bott SRJ, Henderson A, Halls JE, et al. Extensive transperineal template biopsies of prostate: Modified technique and results. Urology. 2006; 68(5):1037-1041.

12. Li H, Yan W, Zhou Y, et al. Transperineal ultrasound-guided saturation biopsies using 11-region template of prostate: report of 303 cases. Urology. 2007;70(6):1157-1161.

13. Merrick GS, Gutman S, Andreini H, et al. Prostate cancer distribution in patients diagnosed by transperineal template-guided saturation biopsy. Eur Urol. 2007;52(3):715-724.

14. Moran BJ, Braccioforte MH, Conterato DJ. Re-biopsy of the prostate using a stereotactic transperineal technique. J Urol. 2006;176(4): 1376-1381.

15. Emiliozzi P, Corsetti A, Tassi B, et al. Best approach for prostate cancer detection: A prospective study on transperineal versus transrectal six-core prostate biopsy. Urology. 2003;61(5):961-966.

16. Vis AN, Boerma MO, Ciatto S, et al. Detection of prostate cancer: A comparative study of the diagnostic efficacy of sextant transrectal versus sextant transperineal biopsy. Urology. 2000;56(4):617-621.

17. Liu IJ, Macy M, Lai Y, et al. Critical evaluation of the current indications for transition zone biopsies. Urology. 2001;57(6):1117-1120.

18. Djavan B, Zlotta A, Remzi M, et al. Optimal predictors of prostate cancer on repeat prostate biopsy: a prospective study of 1,051 men. J Urol. 2000;163(4):1144-1148.

19. Chang JJ, Shinohara K, Hovey RM, et al. Prospective evaluation of systematic sextant transition zone biopsies in large prostates for cancer detection. Urology. 1998;52(1):89-93.

20. Abdel-Khalek M, Sheir KZ, El-Baz M, et al. Is transition zone biopsy valuable in benign prostatic hyperplasia patients with serum prostatespecific antigen $>10 \mathrm{ng} / \mathrm{mL}$ and prior negative peripheral zone biopsy? Scand J Urol Nephrol. 2005;39(1):49-55.
21. Pelzer AE, Bektic J, Berger AP, et al. Are transition zone biopsies still necessary to improve prostate cancer detection? Results from the Tyrol Screening Project. Eur Urol. 2005;48(6):916-921.

22. Reissigl A, Pointner J, Strasser H, et al. Frequency and clinical significance of transition zone cancer in prostate cancer screening. Prostate. 1997;30(2):130-135.

23. Durkan GC, Johnson SP, Hildreth AJ, et al. Improving prostate cancer detection with an extended-core transrectal ultrasonography-guided prostate biopsy protocol. BJU Int. 2002;89(1):33-39.

24. Miyake H, Kurahashi T, Muramaki M, et al. Significance of routine transition zone biopsies in Japanese men undergoing transrectal ultrasound-guided prostate biopsies. Int J Urol. 2005;12(11):964-968.

25. Fink KG, Hutarew G, Esterbauer B, et al. Evaluation of transition zone and lateral sextant biopsies for prostate cancer detection after initial sextant biopsy. Urology. 2003;61(4):748-753.

26. Fleshner NE, Fair WR. Indications for transition zone biopsy in the detection of prostate carcinoma. J Urol. 1997;157(2):556-558.

27. Terris MK, Pham TQ, Issa MM, et al. Routine transition zone and seminal vesicle biopsies in all patients undergoing transrectal ultrasound guided prostate biopsies are not indicated. J Urol. 1997;157(1):204-206.

28. Jones JS, Patel A, Schoenfield L, et al. Saturation technique does not improve cancer detection as an initial prostate biopsy strategy. J Urol. 2006;175(2):485-488.

29. Scattoni V, Raber M, Abdollah F, et al. Biopsy schemes with the fewest cores for detection $95 \%$ of the prostate cancers detected by a 24 -core biopsy. Eur Urol. 2010;57(1):1-8.

30. Epstein JI, Walsh PC, Carter HB. Importance of posterolateral needle biopsies in the detection of prostate cancer. Urology. 2001;57(6): 1112-1116.

31. Falzarano SM, Zhou M, Hernandez AV, et al. Can saturation biopsy predict prostate cancer localization in radical prostatectomy specimens: A correlative study and implications for focal therapy. Urology. 2010;76:682-687.

32. Jeong $\mathrm{CW}, \mathrm{Ku} \mathrm{JH}$, Moon $\mathrm{KC}$, et al. Can conventional magnetic resonance imaging, prostate needle biopsy, or their combination predict the laterality of clinically localized prostate cancer. Urology. 2012;79:1322-1328.

33. Kawakami S, Yamamoto S, Numao N, et al. Direction comparison between transrectal and transperineal extended prostate biopsy for the detection of cancer. Int J Urol. 2007;14:719-724.
Research and Reports in Urology

\section{Publish your work in this journal}

Research and Reports in Urology is an international, peer-reviewed, open access journal publishing original research, reports, editorials, reviews and commentaries on all aspects of adult and pediatric urology in the clinic and laboratory including the following topics: Pathology, pathophysiology of urological disease; Investigation and treatment of

\section{Dovepress}

urological disease; Pharmacology of drugs used for the treatment of urological disease. The manuscript management system is completely online and includes a very quick and fair peer-review system, which is all easy to use. Visit http://www.dovepress.com/testimonials.php to read real quotes from published authors. 